\title{
The Spectrum of the Algebra of Skew Differential Operators and the Irreducible Representations of the Quantum Heisenberg Algebra
}

\author{
Alexander L. Rosenberg \\ Department of Mathematics of Harvard University, Cambridge, MA 02138, USA \\ Received March 12, 1990; in revised form June 17, 1991
}

\begin{abstract}
The left spectrum of a wide class of the algebras of skew differential operators is described. As a sequence, we determine and classify all the algebraically irreducible representations of the quantum Heisenberg algebra over an arbitrary field.
\end{abstract}

\section{Introduction}

The role the Heisenberg algebra plays in mathematical physics (quantum mechanics, quantum field theory, cf. $[\mathrm{J}, \mathrm{C}]$ ) and representation theory (cf. $[\mathrm{K}, \mathrm{D}]$ ) is well known. In the theory of Kac-Moody algebras the Heisenberg algebra provides one of the major tools for construction of irreducible representations (cf. $[\mathrm{FK}])$.

The development of the theory of quantum and classical integrable systems has lead to the notion of Quantum Lie Groups. These objects are certain Hopf algebras created by deformation of universal enveloping algebras of Lie algebras and algebras of functions on Lie groups (cf. [Ji, Dr, FRT, S]). It is natural to expect that the quantum Heisenberg algebra introduced in [FG], which is one of the principal actors of this work, is going to play the same role in the (not yet created) representation theory of quantum groups as the classical Heisenberg algebra does in the representation theory of conventional Lie groups.

The study of the quantum Heisenberg algebra involves naturally a more general class of algebras - the algebras of skew differential operators. These algebras are well known to specialists in ring theory. Here, however, they appear as the objects of noncommutative geometry; and our first concern is to investigate their spectral properties. The means of the investigation are provided by recently introduced local noncommutative algebra (cf. [R1, R2]).

The article is organized as follows. 
In the first section we introduce the notions necessary for the rest of this work and results of the local noncommutative algebra: the left spectrum, Gabriel localizations and its connections with the left spectrum, and the left spectrum of principal ideal domains.

The second section is concentrated around the definition of the algebra of skew differential operators. It contains some important examples and a treatment of skew derivations.

The third, and the most important, section is dedicated to the description of the left spectrum of the algebra of skew differential operators.

The results of the third section enable us to obtain a description of the left spectrum and, in particular, the maximal left spectrum, of the quantum Heisenberg algebra over an arbitrary field. This description is effectuated in Sect. 4. As a sequence, we determine and classify the irreducible representations of the quantum Heisenberg algebra over an arbitrary field. The picture is particularly nice in case of an algebraically closed field.

\section{Preliminaries: Left Spectrum, etc}

1.1 Definition. Let $R$ be an associative ring with unity. Define a preorder $>$ on the set $I_{l} R$ of left ideals of the ring $R$ as follows: $m>n$ if there exists a finite subset $x$ of elements of $R$ such that the left ideal $(m: x):=\{z \in R: z x \subset m\}$ is contained in $n$.

It is easy to see that if the ideal $m$ is two-sided, then the relation $m>n$ is equivalent to the inclusion $m \subseteq n$.

The left spectrum $\operatorname{Spec}_{l} R$ of the ring $R$ consists of all the left ideals $p$, satisfying the following property:

$$
(p: x) \succ p \text { for any } x \in R-p \text {. }
$$

1.2 Note. If every left ideal of the ring $R$ is two-sided then $\operatorname{Spec}_{l} R$ coincides with the completely prime spectrum, $\operatorname{Spec} R$, which, by definition, consists of all the two-sided ideals $p^{\prime}$ such that for any two elements $x, y$ of $R, x y \in p^{\prime}$ if and only if either $x$ or $y$ belongs to $p^{\prime}$.

\subsection{Left Spectrum, Maximal Ideals and Prime Spectrum}

1.3.1 Left Maximal Ideals. For any associative ring $R$, the left spectrum $\operatorname{Spec}_{l} R$ contains the set $\operatorname{Max}_{l} R$ of all the maximal left ideals of $R$.

In fact, if $n, m$ are left ideals of $R$ such that the relation $(m: x) \succ m$ does not hold if $x \in n$, then it does not hold if $x \in m+n$. But, if $m \in \operatorname{Max}_{l} R$ and $n$ is not a subset of $m$, then $m+n=R$. In particular, $m+n$ contains the unity 1 of the ring $R$. Clearly $(m: 1)=m \succ m$. Hence. if $m \in \operatorname{Max}_{l} R$, then $n \subseteq m$.

1.3.2 The Left and Prime Spectra of Left Noetherian Rings and PI Algebras. Recall that the two-sided ideal $p$ of the ring $R$ is prime if, for any pair of elements $x, y$ of $R$, the inclusion $x R y \subset p$, implies that either $x$ or $y$ belongs to the ideal $p$. 
1.3.2.1 Proposition. If the ring $R$ is left noetherian, or if $R$ is a PI-algebra, then $\mathrm{Spec}_{l} R$ contains the set $\mathrm{Spec} R$ of prime ideals of $R$.

Proof. Cf. [R1, Sect. 8.4].

1.4 Left Spectrum and Localizing Filters. Recall that a non-empty set $F$ of left ideals of the ring $R$ is called a localizing filter (or radical filter, or idempotent topologizing filter) if the following conditions hold:

1) for any $m \in F$ and $x \in R$, the left ideal $(m: x)$ belongs to $F$;

2) if $m \in F$ and $n$ is a left ideal of the ring $R$ such that $(n: x) \in F$ for any $x \in m$, then $n$ belongs to $F$.

The importance of localizing filters is due to the fact that they are in one-one correspondence with equivalence classes of localizations of the category $R$-mod of left $R$-modules.

Recall that the localization of an abelian category $\underline{A}$ is an exact functor $Q: \underline{A} \rightarrow \underline{B}$, having fully faithful right adjoint functor.

The correspondence $\{$ localizing filters $\} \leftrightarrow$ \{equivalence classes of the localizations of $R$-mod $\}$ realizes as follows: Let $F$ be a localizing filter; and let $R$-mod/ $F$ denote the full subcategory of the category $R$-mod formed by all the left modules $M$ such that the canonical map $M \rightarrow \operatorname{Hom}_{R}(m, M)$, which sends an element $z \in M$ into the morphism $r \mapsto r z$, is a bijection for any ideal $m$ from the filter $F$. Denote by $H_{F} M$ the direct colimit colim $\left\{\operatorname{Hom}_{R}(m, M): m \in F\right\}$. The $Z$-module $H_{F} M$ has a unique structure of $R$-module such that the map

$$
\eta_{F, M}: M \rightarrow H_{F} M,
$$

induced by the canonical maps $M \rightarrow \operatorname{Hom}_{R}(m, M), m \in F$, is an $R$-module morphism. Moreover, the map $M \mapsto H_{F} M$ is uniquely extended to a functor $H_{F}: R$-mod $\rightarrow R$-mod such that the collection $\eta_{F}:=\left\{\eta_{F, M}: M \in \mathrm{Ob} R-\bmod \right\}$ is a functor morphism $\mathrm{Id} \rightarrow H_{F}$.

Denote the square of the functor $H_{F}$ by $G_{F}$ and call it the Gabriel functor.

1.4.1 Proposition. 1) For any localizing filter $F$, the functor $G_{F}$ takes values in the subcategory $R-\bmod / F$, and the co-restriction of $G_{F}$ onto $R-\bmod / F$ is the localization with the embedding $R-\bmod / F \rightarrow R-\bmod$ as a right adjoint functor.

2) For any localization $Q: R-\bmod \rightarrow B$ the set of all the left ideals $m$ of the ring $R$ such that $Q(R / m)=0$ is a localizing filter, and the category $\underline{B}$ is equivalent to the category $R-\bmod / F_{Q}$.

Proof of this statement can be found in [R1]. It is also deduced without much difficulties from the fundamental results of Gabriel (cf. [G] or [B.D]).

1.4.2 Proposition. a) There is a unique ring structure on $G_{F} R$ for which the canonical $R$-module morphism $j_{F, R}: R \rightarrow G_{F} R$ is a ring morphism.

b) For any $R$-module $M$, there is a unique extension of $R$-module structure on $G_{F} M$ to $G_{F} R$-module structure.

Proof. Cf. [R1, Sect. 2] or [G].

For any left ideal $m$ of the ring $R$, denote by $\langle m\rangle$ the set of all the left ideals $n$ such that the ideal $(n: y)$ is not contained in $m$ for any finite subset $y$ or $R$. 
Note that $\langle m\rangle \subseteq\left\langle m^{\prime}\right\rangle$ if and only if $\left.m^{\prime}\right\rangle m$. In particular, $\langle m\rangle=\left\langle m^{\prime}\right\rangle$ if and only if $m$ and $m^{\prime}$ are equivalent with respect to $\succ$.

The basic property of $\operatorname{Spec}_{l} R$ is the following one:

1.4.3 Proposition. a) If $p \in \operatorname{Spec}_{l} R$ then the set $\langle p\rangle$ is a localizing filter.

b) Let $m$ be a left ideal of the ring $R$ such that the set $\langle m\rangle$ is a localizing filter. Then $m$ is equivalent to an ideal $\rho$ from $\operatorname{Spec}_{l} R:\langle m\rangle=\langle p\rangle$.

Proof. See [R, Proposition 2.2].

1.4.4 Local Categories. Call the abelian category $\underline{A}$ local if

a) every nonzero object $X$ of the category $A$ has a simple subquotient; i.e. there exist two monomorphisms $V \stackrel{i}{\rightarrow} W \rightarrow X$ such that $W / V:=\operatorname{Cok}(i)$ is a simple object;

b) any two simple objects of the category $A$ are isomorphic each other.

It is easy to see that the category of modules over a commutative ring $R$ is local if and only if the ring $R$ is local.

1.4.5 Proposition. For any associative ring $R$ and any $p \in \operatorname{Spec}_{l} R$ the localization $R-\bmod /\langle p\rangle$ of the category $R-\bmod a t\langle p\rangle$ is a local category; and $G_{\langle p\rangle}(R / p)$ is its (unique up to isomorphism) simple object.

Proof can be found in [R1] or [R2].

1.5 Ring Morphisms and Maps of Spectra. The left spectrum does not be have itself functorially with respect to ring morphisms; i.e. if $f: A \rightarrow B$ is a generic ring morphism, then the map $m \mapsto f^{-1}(m)$ does not induce the morphism of spectra. However, we can try to assign to $f$ a map $\sim \operatorname{Spec}_{l} B \rightarrow \sim \operatorname{Spec}_{l} A$ as follows.

Take a left ideal $p$ from $\operatorname{Spec}_{l} B$, set $p^{\prime}:=f^{-1} p$ and consider the set $\Omega_{p^{\prime}}=\left\{\left(p^{\prime}: a\right): a \in A-p^{\prime}\right\}$ of left ideals of the ring $A$. Suppose that the set $\Omega_{p^{\prime}}$ has a maximal element with respect to the preorder $\succ$; i.e. there exists $a \in A-p^{\prime}$ such that if $\left(p^{\prime}: a\right) \succ\left(p^{\prime}: a^{\prime}\right)$ for some $a^{\prime} \in A-p^{\prime}$ then $\left(p^{\prime}: a\right) \approx\left(p^{\prime}: a^{\prime}\right)$.

In particular, if $x \in A-\left(p^{\prime}: a\right)$ (or, equivalently, $\left.x a \in A-p^{\prime}\right)$ then $\left(p^{\prime}: a\right) \approx\left(p^{\prime}: x a\right)=$ $\left(\left(p^{\prime}: a\right): x\right)$; i.e. $\left(p^{\prime}: a\right) \in \operatorname{Spec}_{l} A$.

Note now that $\left(p^{\prime}: a\right):=\left(f^{-1} p: a\right)=f^{-1}(p: f(a))$. Since, by condition, $p \in \operatorname{Spec}_{l} B$ and $f(a) \notin p$, the left ideal $(p: f(a))$ is equivalent to $p$.

1.5.1 Remarks. 1) Suppose the left ideal $p$ of the $\operatorname{ring} B$ satisfies the condition: $(p: r) \subseteq p$ for any $r \in B-p$ (it is natural to call such ideals completely prime). Then, for any $a \in A-f^{-1}(p)$ we have:

$$
\left(f^{-1}(p): a\right)=f^{-1}(p: f(a)) \subseteq f^{-1}(p) .
$$

In words, it means that all the ideals of the set $\Omega_{f}{ }^{-1}(p)$ are equivalent one to another. In particular, $f^{-1}(p) \in \operatorname{Spec}_{l} A$.

2) The most obvious sufficient condition of the existence of maximal elements in the set $\Omega_{p}$, is the following one:

(i) The preordered set $\left(I_{l} A, \succ\right)$ is noetherian.

If the ring $A$ is commutative (more generally, if all the left idals of $A$ are two-sided) then condition (i) is the same as

(ii) $A$ is a noetherina ring. 
3) Even if $\operatorname{Max} \Omega_{f^{-1}(p)}$ is nonempty, the map

$$
p \mapsto p^{\prime}=\left(f^{-1} p: a\right) \in \operatorname{Spec}_{l} A
$$

is not, in general, well defined with respect to $\succ$; i.e. it may be that two ideals from $\operatorname{Spec}_{l} B$ are equivalent, but their "preimages" in $\operatorname{Spec}_{l} A$ are not.

However, this construction provides one of the main tools for studying left spectra of rings.

The other major instrument is a properly chosen localization. Any localization functor $Q=Q_{F}: R-\bmod \rightarrow R-\bmod / F$ sends the ideals from $\operatorname{Spec}_{l} R-F$ into the ideals from $\operatorname{Spec}_{l} G_{F} R$. And often (for an appropriate $R$ ) the left spectrum of localized ring, $\operatorname{Spec}_{l} G_{F} R$, is much easier to describe than $\operatorname{Spec}_{l} R$. The most favorable situation is when $G_{F} R$ turns out to be a left and right principal domain.

1.6 The left Spectrum of a Principal Domain. Let $R$ be left and right principal ideal domain. I.e. $R$ is a ring without zero-divisors such that each left and right ideal of $R$ is generated by one element.

1.6.1 Proposition. Let $R$ be a left and right principal domain. Then every nonzero ideal from $\operatorname{Spec}_{l} R$ is equivalent to a left maximal ideal. Every left maximal ideal of the ring $R$ is of the form $R f$, where $f$ is an irreducible element of the ring $R$.

Proof. Let $p \in \operatorname{Spec}_{l} R$. Since $R$ is a left principal ideal domain, $p=R f$ for some element $f \in R$. It is easy to see that the absence of zero-divisors guarantees that the right ideal $f R$ is proper.

In fact, if $f g=1$ then $(1-g f) g=g(1-f g)=0$; therefore, $g f$ is also equal to 1; i.e. $p=R f=R$.

Being a proper ideal, $f R$ is contained in a right maximal ideal $\mu$. Since $R$ is a right principal ideal domain, $\mu=g R$ for some irreducible element $g$ of the ring $R$. The inclusion $f R \subseteq g R$ means that $f=g h$ for some $h$. Note that $h \notin p$.

Indeed,

$$
[h \in p] \Leftrightarrow\left[h=h^{\prime} f \text { for some } f \in R\right] \Leftrightarrow\left[g h^{\prime}=1\right] \Leftrightarrow[\mu=g R=R] .
$$

Since $p \in \operatorname{Spec}_{l} R$ and $h \notin p$, the left ideal $(p: h)$ is equivalent to $p$. Clearly $R g \subseteq(p: h)$. But $R g$ is a maximal left ideal (thanks to the irreducibility of $g$ ); hence $R g=(p: h)$.

1.6.2 Lemma. Let $R$ be a left principal domain. Then every localizing filter of left ideals is of the form

$$
F_{S}:=\left\{m \in I_{l} R: m \cap S \neq \varnothing\right\}
$$

for some Ore multiplicative subset $S$.

Proof. Let $F$ be a localizing filter of left ideals. Denote by $S$ the set of all the elements $t \in R$ such that $R t \in F$. Since $R$ is a left ideal principal ring, $(R t: x)=R t^{\prime}$; i.e. for any $t \in S$ and any $x \in R$ there exist $y \in R$ and $t^{\prime} \in S$ such that $t^{\prime} x=y t$. The second Ore condition - if $s a=0$ for some $s \in S$ then there exists $s^{\prime} \in S$ such that $a s^{\prime}=0$ - holds automatically.

It is rest to show that if $s, t \in S$ then $s t \in S$; i.e. $R s t \in F$. 
In fact, for any element $x=a t$ of the left ideal $R t$, we have:

$$
(R s t: x)=((R s t: t): a) \supseteqq(R s: a) .
$$

Since $(R s: a) \in F$ for any $a \in R$, then $(R s t: x) \in F$ for any $x \in R t$. Therefore $R s t \in F$.

\section{The Algebra of Skew Differential Operators. Skew Derivations}

2.1 Definition. Let $A$ be a commutative algebra with unity over a field $k$; let $\vartheta$ be an automorphism and $d$ a $\vartheta$-derivation of $A$; i.e. $d$ is a $k$-linear map $A \rightarrow A$ such that

$$
d(a b)=d(a) b+\vartheta(a) d(b) .
$$

The associative ring $A[x ; \vartheta, d]$ of $\vartheta$-skew differential operators is a left $A$-module of polynomials in $x$ with coefficients in $A$ and with the multiplication, which is uniquely defined by the property:

$$
x a=\vartheta(a) x+d(a) \text { for any } a \in A .
$$

2.2 Example. The algebra $A[x ; \vartheta, 0]$ coincides with the algebra $[A[x ; \vartheta]$ of skew polynomials over $A$.

2.3 Example. Let $A=k[y]$. Then the algebra $A\left[x ; \mathrm{Id},{ }^{\prime}\right]$, where ' is the usual derivation in $k[y]$, is the first Weyl algebra $A_{1}(k)$.

In fact, the relations (2) are equivalent to the relation

$$
x y=y x+1 .
$$

2.4 Example. Quantum Heisenberg Algebra $H_{q, h}$. Let $A=k[y]$, and let $\vartheta$ be a generic automorphism of the $k$-algebra $k[y]$; i.e. $\vartheta(y)=q y+\alpha$ for some $q, \alpha \in k$.

The condition ' $d$ is a $\vartheta$-derivation of $k[y]$ ' implies that

$$
d\left(y^{n}\right)=\left(\sum_{1 \leqq i \leqq n} y^{n-i} \vartheta(y)^{i-1}\right) h,
$$

where $h=d y$. It is easy to verify that any choice of the polynomial $h$ determines a $\vartheta$-derivation of the algebra $k[y]$. So we fix $h \in k[y]$ and denote the corresponding algebra $k[y][x ; \vartheta, d], d y=h(y)$, by $A_{1}(\vartheta, h)$. In other words, $A_{1}(\vartheta, h)$ is the $k$-algebra with generators $x, y$ and the determining relations

$$
x y-(\vartheta(y) x=h(y) \text {. }
$$

Clearly $A_{1}(\mathrm{Id}, 1)$ is a first Weyl algebra.

If $\vartheta f(y)=f(q y)$ for some $q \neq 0$ and $\operatorname{deg}(h)=0$ then $A_{1}(\vartheta, h)$ is a so-called $q$-Heisenberg algebra. It will be denoted by $H_{q, h}$.

2.5 The Skew Derivations of Algebras and of Their Algebras of Fractions. Let $S=S(A)$ denote the set of all the non-zero divisors of the algebra $A$. Clearly any automorphism $\vartheta$ of the algebra $A$ induces a bijection $S \rightarrow S$. This implies that the automorphism $\vartheta$ extends (uniquely) up to an automorphism of the algebra Fract $(A):=S^{-1} A$, which we denote by the same letter. 
2.5.1 Lemma. Any $\vartheta$-derivation d of the algebra $A$ has a unique extension up to a $\vartheta$-derivation of the algebra $\operatorname{Fract}(A)$.

Proof. In fact, if $d^{\sim}$ is an extension of a $\vartheta$-derivation $d$ on $\operatorname{Fract}(A)=S^{-1} A$ then, for any $a \in A$ and $s \in S$, we have:

$$
d(a)=d^{\sim}((a / s) s)=d^{\sim}(a / s) s+\vartheta(a / s) d(s)
$$

or, equivalently,

$$
d^{\sim}(a / s)=d(a) / s-\vartheta(a) d(s) /(s \vartheta(s)) .
$$

The equality (1) shows that the extension $d^{\sim}$ is uniquely determined by $d$, and, at the same time, provides the formula for this extension.

Therefore, it remains to verify that the map $d^{\sim}$, defined by (1), is a $\vartheta$-derivation of $\operatorname{Fract}(A)$ for any $\vartheta$-derivation $d$.

It follows from (1) that, for any $s \in S$,

$$
d^{\sim}(1 / s)=-d(s) /(s \vartheta(s)) .
$$

We have:

$$
\begin{aligned}
d^{\sim}(1 /(s t)) & =-d(s t) /(s t \vartheta(s t))=-\{d(s) t+\vartheta(s) d(t)\} /(s t \vartheta(s t))) \\
& =-\{d(s) /(s \vartheta(s))\} \vartheta(1 / t)-\{d(t) /(t \vartheta(t))\}(1 / s) \\
& =d^{\sim}(1 / t)(1 / s)+\vartheta(1 / t) d^{\sim}(1 / s) .
\end{aligned}
$$

Set for convenience $x=1 / s, y=1 / t$. Then it follows from (2) and (3) that

$$
\begin{aligned}
d(a b \cdot x y) & =d(a b) x y+\vartheta(a b) d(x y)=\{d(a) b+\vartheta(a) d(b)\} x y+\vartheta(a b)\{d(x) y+\vartheta(x) d(y)\} \\
& =d(a) b x y+\vartheta(a) d(b) x y+\vartheta(a b) d(x) y+\vartheta(a b) \vartheta(x) d(y) \\
& =\{d(a) b x y+\vartheta(a b) \vartheta(x) d(y)\}+\vartheta(a)\{d(b) x+\vartheta(b) d(x)\} y \\
& =\{d(a) b x y+\vartheta(a b) \vartheta(x) d(y)\}+\vartheta(a) d(b x) y .
\end{aligned}
$$

On the other hand,

$$
\begin{aligned}
d(a x) b y+\vartheta(a x) d(b y) & =\{d(a) x+\vartheta(a) d(x)\} b y+\vartheta(a x)\{d(b) y+\vartheta(b) d(y)\} \\
& =d(a) b x y+\vartheta(a) d(x) b y+\vartheta(a x) d(b) y+\vartheta(a x) \vartheta(b) d(y) \\
& =\{d(a) b x y+\vartheta(a b) \vartheta(x) d(y)\}+\vartheta(a)\{d(x) b+\vartheta(x) d(b)\} y \\
& =\{d(a) b x y+\vartheta(a b) \vartheta(x) d(y)\}+\vartheta(a) d(b x y) y .
\end{aligned}
$$

This shows that

$$
d^{\sim}((a / s)(b / t))=d^{\sim}(a / s)(b / t)+\vartheta(a / s) d(b / t),
$$

i.e. $d^{\sim}$ is a $\vartheta$-derivation of the algebra $\operatorname{Fract}(A)$.

2.6 The Simplest $९$-Derivation. It is easy to see that the map

$$
\partial_{\vartheta}: a \mapsto a-\vartheta(a), \quad a \in A,
$$

is a $\vartheta$-derivation:

$$
\partial_{\vartheta}(a b):=a b-\vartheta(a b)=(a-\vartheta(a)) b+\vartheta(a)(b-\vartheta(b)):=\partial_{\vartheta}(a) b+\vartheta(a) \partial_{\vartheta}(b) .
$$


In the classical case, $\vartheta=\mathrm{Id}$, (and only in the classical case) the $\vartheta$-derivation $\partial_{\vartheta}$ is trivial. But if, on the contrary, the difference between $\vartheta$ and Id is such that there exists an element $u$ such that $u-\vartheta(u)$ is not a zero divisor, then, as we shall see in a moment, the $\vartheta$-derivation $\partial_{\vartheta}$ is universal in the following sense: every $\vartheta$-derivation of the algebra $A$ is equal to $g \partial_{\vartheta}$ for a unique element $g$ of $\operatorname{Fract}(A)$.

2.7 A Formula for $\vartheta$-Derivation. Let $d$ be an arbitrary $\vartheta$-derivation of the algebra $A$. Since $A$ is commutative, we have the following equations:

$$
d(a) b+\vartheta(a) d(b)=d(a b)=d(b a)=d(b) a+\vartheta(b) d(a)
$$

which imply that

$$
d(a)(b-\vartheta(b))=d(b)(a-\vartheta(a)) .
$$

Therefore, if $\vartheta(b)-b$ is not a zero divisor, we can embed the algebra $A$ into its algebra of fractions, Fract $(A)$, (or, at least, into the localization of $A$ at multiplicative subset $(\vartheta(b)-b)$ generated by the element $\vartheta(b)-b$, and rewrite the equality (1) in the following form:

$$
d(a)=d(b)\left(b-\vartheta(b)^{-1}(a-\vartheta(a)) .\right.
$$

2.7.1 Lemma. Let $u$ be an element of the algebra Fract $(A)$ such that the element $u-\vartheta(u)$ is invertible.

Then every $\vartheta$-derivation of the quotient algebra $Q A$ is of the form

$$
d(a)=h\left(u-\vartheta(u)^{-1}(a-\vartheta(a)),\right.
$$

where $h=d u$ may be any element of $\operatorname{Fract}(A)$.

Proof. If $h=d u$ then the formula (3) coincides with (2).

Since $a \mapsto h d(a)$ is a $\vartheta$-derivation for any $\vartheta$-derivation $d$ and for any element $h$, and the map $\partial_{\vartheta}, \partial_{\vartheta}(a):=a-\vartheta(a)$, is a $\vartheta$-derivation (cf. 2.6), then the map

$$
\partial_{\vartheta, u}: a \mapsto(u-\vartheta(u))^{-1}(a-\vartheta(a))
$$

is a Э-derivation.

2.7.2 Corollary. Let the $\vartheta$ be an automorphism of the algebra $A$ such that there exists an element $u$ of $A$, for which $\partial_{\vartheta}(u):=u-\vartheta(u)$ is a non-zero divisor. Then each $\vartheta$-derivation $d$ is equal to $g \partial_{\vartheta}: a \mapsto g(a-\vartheta(a))$ for a unique element $g$ of $\operatorname{Fract}(A)$.

2.7.3 Corollary. Let $u$ be an element of the algebra $A$ such that

1) $u-\vartheta(u)$ is a non-zero divisor;

2) for any element $a \in A$ there exists an element $\partial_{\vartheta, u}(a)$ of $A$ such that $a-\vartheta(a)=\partial_{\vartheta, u}(a)(u-\vartheta(u))$.

Then the map $\partial_{\vartheta, u}: a \mapsto \partial_{\vartheta, u}(a)$ is a $\vartheta$-derivation; and any $\vartheta$-derivation of the algebra $A$ is equal to $h \partial_{\vartheta, u}$ for some unique element $h$ of $A$.

2.7.4 Example. q-derivations. Let $q \in k, q \neq 0,1, A=k[y]$ and $\vartheta=q_{*}: f(y) \mapsto f(q y)$ for any $f \in k[y]$. Take $u:=y$. Then $u-\vartheta(u)=y(1-q)$. Obviously the conditions of Corollary 2.7.3 are satisfied and the $\vartheta$-derivation $\partial_{\vartheta, u}=\partial_{q, y}$ is defined by the formula:

$$
\partial_{q, y} f(y)=(f(y)-f(q y)) /(y(1-q)) .
$$


According to Corollary 2.7.3, every $q$-derivation $d$ is equal to $h(y) \partial_{q, y}$ for a unique polynomial $h(y)$.

\section{The Left Spectrum of the Algebra of Skew Differential Operators}

We assume that $A$ is a commutative noetherian domain. We also suppose that $d(a)=0$ if and only if $a \in k$.

Fix an ideal $p$ from $\operatorname{Spec}_{l} A[x ; \vartheta, d]$. Replacing, if necessary, the ideal $p$ by an equivalent ideal $(p: a)$, where $a$ is an element of $A-p$ such that $(p: a) \cap A$ is a maximal element of the set $\left\{\left(p: a^{\prime}\right) \cap A: a^{\prime} \in A-p\right\}$, we assume that $p \cap A \in \operatorname{Spec} A$ (cf. 1.5).

Consider the possibilities.

(a) $p \cap A=\{0\}$. Note that this condition implies that $A$ is a prime ring. The localization $Q$ at the set $A-\{0\}$ sends the algebra $A[x ; \vartheta, d]$ into the algebra $Q A[x ; \vartheta, d]$ over the skew field of quotients $Q A$, and the ideal $p$ into the left ideal $Q p$ from $\operatorname{Spec}_{l} Q A[x ; \vartheta, d]$.

The ring $Q A[x ; \vartheta, d]$ is a left and right principal ideal domain (since it is euclidean). Therefore $Q p$ is a left principal ideal generated by an irreducible element $b=b_{p}$ of the ring $A[x ; \vartheta, d]$. The ideal $p$ coincides with $A \cap Q p=A \cap Q A[x ; \vartheta, d] b_{p}$.

(b) Suppose now that the ideal $p^{\prime}=p \cap A$ is nonzero.

(b1) Let $p^{\prime}$ is invariant with respect to the automorphism $\vartheta$ and to the derivation $d$. Then we can take quotient algebra $A[x ; \vartheta, d] / A[x ; \vartheta, d] p^{\prime} \cong A^{\prime}[x ; \vartheta, d]$, where $A^{\prime}:=A / p^{\prime}$. The image $p^{\prime \prime}$ of the ideal $p$ in $A^{\prime}[x ; \vartheta, d]$ has the trivial intersection with $A^{\prime}$. Therefore either $p^{\prime \prime}=\{0\}$ (i.e. $p=A[x ; \vartheta, d] p^{\prime}$ ) or $p^{\prime \prime}=A[x ; \vartheta, d] \cap Q A^{\prime}[x ; \vartheta, d] b_{p}$ for some irreducible element $b_{p}$ of the principal ideal domain $Q A^{\prime}[x ; \vartheta, d]$.

(b2) Suppose that $p^{\prime}$ is invariant with respect to the automorphism $\vartheta$, but is not invariant with respect to $d$.

3.1 Lemma. For any positive integer $n$ and for any $a \in A$,

$$
\vartheta^{n}(a) x^{n}=x^{n} a-\sum_{0 \leqq i \leqq n-1} x^{n-i-1} d\left(\vartheta^{i}(a)\right) x^{i}
$$

Proof. Equation (1.1) is equivalent to the determining Eq. (2) in 2.1. Assuming that (1.n) holds, we have:

$$
\begin{aligned}
\vartheta^{n+1}(a) x^{n+1}= & \left(x^{n} \vartheta(a)-\sum_{0 \leqq i \leqq n-1} x^{n-i-1} d\left(\vartheta^{i+1}(a)\right) x^{i}\right) x=x^{n}(x a-d a) \\
& -\sum_{0 \leqq i \leqq n-1} x^{n-i-1} d\left(\vartheta^{i+1}(a)\right) x^{i+1}=x^{n+1} a-\sum_{0 \leqq i \leqq n} x^{n-i} d\left(\vartheta^{i}(a)\right) x^{i} .
\end{aligned}
$$

3.2 Lemma. Suppose that the ideal $p^{\prime}=p \cap A$ is invariant with respect to $\vartheta$; but there exists a positive integer $N$, satisfying the following condition:

For any integer $n, 1 \leqq n \leqq N$, an $r \in p^{\prime}$ can be found such that

$$
\sum_{0 \leqq i \leqq n-1} \vartheta^{-i} d\left(\vartheta^{i}(r) \notin p^{\prime}\right.
$$

Then, for any element $g(x)=x^{m} a+x^{m-1} b+\cdots$ from $A[x ; \vartheta, d]$ such that $a \in A-p$ and $n \leqq N$, there exists an element $u$ of the algebra $A$, for which $u g \in(A+p)-p$. 
Proof. 1) Let $\operatorname{deg}(g)=1$; i.e. $g(x)=x a+b$ for some elements $a, b$ of $A$. By condition (1) (for $n=1$ ) there exists $r \in p^{\prime}$ such that $d r \notin p^{\prime}$. We have

$$
\vartheta(r) g(x)=x r a+d r+\vartheta(r) b \in d r+p \text {. }
$$

2) Suppose that the statement is true for polynomials of degree less than $n, n \leqq N$; and let $g(x)=x^{n} a+x^{n-1} b+\cdots$ be a polynomial of degree $n$ such that ac $A-p^{\prime}$.

By hypothesis, an element $r \in p^{\prime}$ can be found such that

$$
\sum_{0 \leqq i \leqq n-1} \vartheta^{-i} d\left(\vartheta^{i}(r)\right) \notin p^{\prime}
$$

It follows from Lemma 3.1 that

$$
\vartheta^{n}(r) g(x)=x^{n} r a+x^{n-1}\left(\sum_{0 \leqq i \leqq n-1} \vartheta^{-i} d\left(\vartheta^{i}(r)\right) a+x^{n-1} \vartheta(r) b+h(x)\right),
$$

where $h(x)$ is a polynomial of degree less than $n-1$.

Since $r a$ and $\vartheta(r) b$ are elements of $p$, we can write:

$$
\vartheta^{n}(r) g(x)=\left(x^{n-1}\left(\sum_{0 \leqq i \leqq n-1} \vartheta^{-i} d\left(\vartheta^{i}(r)\right)+h(x)\right) \bmod p .\right.
$$

Thanks to the condition (1), we can find, by induction hypothesis, an element $v \in A$ such that

$$
v \vartheta^{n}(r) g(x) \in(A+p)-p
$$

3.3 Corollary. Suppose that the ideal $p^{\prime}=p \cap A$ is invariant with respect to $\vartheta$; and for any integer $n \geqq 1$ there exists $r \in p^{\prime}$ such that

$$
\sum_{0 \leqq i \leqq n-1} \vartheta^{-i} d\left(\vartheta^{i}(r)\right) \notin p^{\prime}
$$

Then the ideal $p$ is generated by $p^{\prime}:=p \cap A$; i.e. $p=A[x ; \vartheta, d] p^{\prime}$.

3.4 Proposition. Suppose that $p^{\prime}$ is a prime ideal of the algebra $A$ satisfying the following conditions:

(a) $p^{\prime}$ is invariant with respect to $\vartheta$;

(b) for any integer $n \geqq 1$ there exists $r \in p^{\prime}$ such that

$$
\sum_{0 \leqq i \leqq n-1} \vartheta^{-i} d\left(\vartheta^{i}(r)\right) \notin p^{\prime}
$$

Then

1) The left ideal $p=A[x ; \vartheta, d] p^{\prime}$ belongs to the left spectrum of the algebra $A[x ; \vartheta, d]$.

2) If the ideal $p^{\prime}$ is maximal then the left ideal $p=A[x ; \vartheta, d] p^{\prime}$ is maximal.

Proof. 1) Note that the only hypothesis used in the argument proving Lemma 3.2 are the conditions on the ideal $p^{\prime}$, the same as here, and the equality $p^{\prime}=p \cap A$. Therefore the statement of Lemma 3.2 holds for the left ideal $p=A[x ; \vartheta, d] p^{\prime}$.

Let $g=g(x)$ be an element from $A[x ; \vartheta, d]-p$. We have to show that the left ideal $(p: g)$ is equivalent to $p$. 
By Lemma 3.2 there exists an element $u \in A$ such that $u g \in(A+p)-p$; i.e. $u g(x)=a+f(x)$, where $a \in A-p^{\prime}$ and $f(x) \in p$. Therefore we have:

$$
((p: g): u)=(p: u g)=(p: a+f)=(p: a) .
$$

Note now that $\left(p^{\prime}: a\right)=p^{\prime}$, since the ideal $p^{\prime}$ is prime and $a \in A-p^{\prime}$, and, therefore,

$$
(p: a):=\left(A[x ; \vartheta, d] p^{\prime}: a\right)=A[x ; \vartheta, d]\left(p^{\prime}: a\right)=A[x ; \vartheta, d] p^{\prime}:=p .
$$

Thus, $((p: g): u)=p$. In particular, $p$ and $(p: g)$ are equivalent each other.

2) Suppose now that the ideal $p^{\prime}$ is maximal. Let $g(x)$ be an arbitrary element from $A[x ; \vartheta, d]-p$. We have to show that the left ideal $\mu=p+A[x ; \vartheta, d] g$ coincides with $A[x ; \vartheta, d]$.

In fact, there exists $u \in A$ such that $u g(x)=a+f(x)$ for some $a \in A-p^{\prime}$ and $f(x) \in p$. Since $u g(x)$ and $f(x)$ belong to the ideal $\mu$, the element $a$ also belongs to $\mu$. But then $\mu$ contains $p^{\prime}+A a$; and $p^{\prime}+A a=A$ thanks to the maximality of $p^{\prime}$. In particular, $\mu$ contains the unity element of $A[x ; \vartheta, d]$.

3.5 Proposition. Suppose that $p^{\prime}=p \cap A$ is a prime ideal of the algebra A satisfying the following conditions:

(a) $p^{\prime}$ is invariant with respect to $\vartheta$;

(b) for any integer $n \geqq 1$ there exists $r \in p^{\prime}$ such that

$$
\sum_{0 \leqq i \leqq n-1} \vartheta^{-i} d\left(\vartheta^{i}(r)\right) \notin p^{\prime}
$$

Then

1) The quotient module $V^{p^{\prime}}:=A[x ; \vartheta, d] / p$ is the direct sum of its $k$-submodules $V_{i}=x^{i} V_{0}, i \geqq 0$, where $V_{0}$ is the image of $A$ with respect to the canonical epimorphism $A[x ; \vartheta, d] \rightarrow V^{p^{\prime}}$.

The action of $A[x ; \vartheta, d]$ is given by the formulas:

$$
x \cdot\left(x^{i} v\right)=x^{i+1} v ; \quad a \cdot\left(x^{i} v\right)=\sum_{0 \leqq j \leqq i} x^{j} \varphi_{i, j}(a) \cdot v
$$

for any $v \in V_{0}, a \in A$ and $i \geqq 1$, where the functions $\varphi_{i, j}($ from $A$ to $A$ ) are determined by the relations:

$$
\begin{aligned}
\varphi_{0,0}(a) & \left.=a ; \quad \varphi_{i, 0}(a)=\left(-d \circ \vartheta^{-1}\right)^{i}(a)\right) \\
\varphi_{i, j}(a) & =\varphi_{i-1, i-1}\left(\vartheta^{-1}(a)\right) \quad \text { for } \quad i \geqq 1 \\
\varphi_{i, j}(a) & =\varphi_{i-1, j-1}\left(\vartheta^{-1}(a)\right)-\varphi_{i-1, j}\left(d\left(\vartheta^{-1}(a)\right)\right)
\end{aligned}
$$

for $i \geqq 1,0 \leqq j \leqq i-1$.

2) The following properties of the $A[x ; \vartheta, d]$-module $V^{p^{\prime}}$ are equivalent:

(i) The $A[x ; \vartheta, d]$-module $V^{p^{\prime}}$ is irreducible.

(ii) The A-module $V_{0}$ is irreducible.

(iii) The ideal $p^{\prime}$ of the algebra $A$ is maximal.

Proof. 1) The formulas (2), which follow from the relations:

$$
a \cdot\left(x^{i} v\right)=x \vartheta^{-1}(a) x^{i-1} v-d\left(\vartheta^{-1}(a)\right) x^{i-1} v
$$

show that the sum of $k$-submodules (subspaces) $V_{i}:=x^{i} V_{0}, i \geqq 0$, is a submodule of the $A[x ; \vartheta, d]$-module $V^{p^{\prime}}$. 
Now, suppose that $\sum_{0 \leqq i \leqq m} x^{i} v_{i}=0$ for some elements $v_{i}$ of the subspace $V_{0}$. Every element $v_{i}$ can be written as $a_{i} u_{0}$, where $a_{i} \in A$ and $u_{0}$ is the image of the unity of $A[x ; \vartheta, d]$. Thus, the equality $\sum_{0 \leqq i \leqq m} x^{i} v_{i}=0$ means exactly that the element $\sum_{0 \leqq i \leqq m} x^{i} a_{i}$ of the algbera $A[x ; \vartheta, d]$ belongs to the ideal $p$. But, according to Corollary $3.3, p=A[x ; \vartheta, d] p^{\prime}$; in particular, all the coefficients $a_{i}, 0 \leqq i \leqq m$, belong to $p^{\prime}=p \cap A$. Since $p^{\prime}$ is the annihilator of the element $u_{0}$, this means that all the elements $v_{i}, 0 \leqq i \leqq m$, are zeros.

2) Evidently, (ii) $\Leftrightarrow$ (iii) since the $A$-modules $V_{0}$ and $A / p^{\prime}$ are canonically isomorphic each other.

(i) $\Rightarrow$ (ii). For any nonzero proper submodule $W$ of the $A$-module $V_{0}$, the submodule $A[x ; \vartheta, d] W$ of $V^{p^{\prime}}$ is also nonzero and proper.

In fact, it follows from the formulas (3) (cf. the heading 1) of this proposition) that $A[x ; \vartheta, d] W \cap V_{0}=W$.

(ii) $\Rightarrow$ (i). Let $w$ be a nonzero element of $V^{p^{\prime}}$. We have to show that, provided $V_{0}$ is an irreducible $A$-module, $A[x ; \vartheta, d] \cdot w=V^{p^{\prime}}$.

Clearly $w$ is the image of polynomial

$$
g(x)=x^{m} a+x^{m-1} b+\cdots
$$

from $A[x ; \vartheta, d]$ such that $a \in A-p=A-p^{\prime}$. By Lemma 3.2 there exists an element $u \in A$ such that $u g \in(A+p)-p$. This means that $u \cdot w$ is a nonzero element of the image $V_{0}$ of the algebra $A$ in $V^{p^{\prime}}$. Since, by condition, the $A$-module $V_{0}$ is irreducible, there exists $a \in A$ such that $a u \cdot w$ is the image of the unity of $A[x ; \vartheta, d]$. This means that

$$
A[x ; \vartheta, d] w \supseteqq A[x ; \vartheta, d] a u \cdot w=V^{p^{\prime}}
$$

3.6 Example. q-Heisenberg Algebra. Let $A=k[y]$, and let $\vartheta$ be a generic automorphism of $A$; i.e. $(\vartheta f)(y)=f(q y+\alpha)$ for some $q$ and $\alpha$ from $k, q \neq 0$. If $\alpha \neq 0$; then there are no $\vartheta$-invariant ideals. Therefore we consider the case when $\alpha=0$; i.e. when the algebra $k[y][x ; \vartheta, d]$ coincides with the $q$-Heisenberg algebra $H_{q, h}=H_{q, h}[x, y]$, where $h=d(y)$ (cf. Example 2.4).

If $q$ is not a root of unity, then the only $\vartheta$-invariant prime ideal in $k[y]$ is $k[y] y$.

As we have seen (cf. Example 2.4.3), any $\vartheta$-derivation of $k[y]$ is uniquely determined by $d y=h(y)$ (cf. Example 2.7.4):

$$
d f(y)=h(y) \partial_{y} f(y)=h(y)(f(y)-f(q y))(y(1-q))^{-1} .
$$

In particular,

$$
d\left(y^{n}\right)=\left(\sum_{1 \leqq i \leqq n} y^{n-i} \vartheta(y)^{i-1}\right) h=y^{n-1} h(y)\left(1-q^{n}\right) /(1-q) .
$$

It follows from (2) that the condition $\sum_{0 \leqq i \leqq n-1} \vartheta^{-i} d\left(\vartheta^{i}(r)\right) \notin k[y] y$ for some $r \in k[y] y$ means that $\sum_{0 \leqq i \leqq n-1} \vartheta^{-i} d\left(\vartheta^{i}(y)\right) \notin k[y] y$. But

$\sum_{0 \leqq i \leqq n-1} \vartheta^{-i} d\left(\vartheta^{i}(y)\right)=\sum_{0 \leqq i \leqq n-1} \vartheta^{-i} d\left(q^{i} y\right)=\sum_{0 \leqq i \leqq n-1} q^{i} \vartheta^{-i} h(y)=\sum_{0 \leqq i \leqq n-1} q^{i} h\left(q^{-i} y\right)$. 


$$
\begin{aligned}
& \text { So, if } h(y)=\sum_{0 \leqq i \leqq m} h_{i} y^{i} \text {, then } \\
& \qquad \sum_{0 \leqq i \leqq n-1} q^{i} h\left(q^{-i} y\right)=h(0)\left(1-q^{n}\right) /(1-q)+\sum_{1 \leqq i \leqq m} h_{i} y^{i}\left(1-q^{-n(i-1)}\right) /\left(1-q^{-i}\right) .
\end{aligned}
$$

In particular, if $q$ is not a root of unity then the condition $\sum_{0 \leqq i \leqq n-1} \vartheta^{-i} d\left(\vartheta^{i}(y)\right) \notin k[y] y$ holds if and only if $d(y)(0)=h(0) \neq 0$.

Note that in case $q=1$ (as well as in the general situation, when $\vartheta=$ Id) it is necessary to require that $\operatorname{Char}(k)=0$, since then $\sum_{0 \leqq i \leqq n-1} \vartheta^{-i} d\left(\vartheta^{i}(y)\right)=n d(y)=n h(y)$.

By Proposition 3.5, the representation $V^{(y)},(y)=k[y] y$, of the $q$-Heisenberg algebra $H_{q, h}$ is irreducible. We can describe it more precisely. Suppose for simplicity that $\operatorname{deg}(h)=0$; i.e. $d(y)=h \in k$.

Note that $V_{0}$ is isomorphic to the field $k$ (as a $k[y]$-module). Therefore $V^{(y)}=\sum_{i \geqq 0} x^{i} V_{0}$ is isomorphic, as a $k$-module, to $k[x]$.

It follows from Lemma 3.1 that the transfered from $V^{(y)}$ action of $k[y]$ on $k[x]$ is defined as follows:

$$
y \cdot x^{n}=x^{n-1} h\left(1-q^{-n}\right) /(1-q) .
$$

It is easy to see that the right part of (3) can be written as the operator $-(h / q) \partial_{1 / q}\left(x^{n}\right)$, where $\partial_{1 / q}$ is a $1 / q$-derivation (cf. Example 2.7.4). Recall that the $1 / q$-derivation is defined by the formula:

$$
\partial_{1 / q}(f)(x)=x^{-1}\left(f\left(q^{-1} x\right)-f(x)\right) /\left(q^{-1}-1\right) .
$$

It is advisable not to mix the generators of the algebra $H_{q, h}$ with the description of the spaces of the representations of $H_{q, h}$. Thus we say that the canonical representation $V^{(y)}$ is realized as the $k$-module $k[z]=\left\{\sum z^{m} a_{m}: a_{m} \in k\right\}$ of polynomials in $z$, where $x$ acts by the multiplication and $y$ by the $1 / q$-derivation:

$$
(x \cdot f)(z)=z f(z), \quad(y \cdot f)(z)=-h / q \partial_{1 / q} f(z) .
$$

Clearly, when $q=1$ the representation (5) becomes the canonical representation of the Weyl algebra:

$$
(x \cdot f)(z)=z f(z), \quad(y \cdot f)(z)=-h \frac{d f}{d x}(z)
$$

3.6.1 The Conjugate q-Heisenberg Algebra. It is seen from determining the quantum Heisenberg algebra relations

$$
x y-q y x=h
$$

that in the conventional case, when $h \in k$, we can change places $x$ and $y$. More explicitly, the correspondence $x \mapsto \hat{y}, y \mapsto x^{\wedge}$ determines an isomorphism $\sigma$ of the quantum Heisenberg algebra $H_{q, h}=H_{q, h}[x, y]$ onto $H_{1 / q,-h / q}=H_{1 / q,-h / q}\left[x^{\wedge}, y^{\wedge}\right]$.

Obviously the inverse to $\sigma$ isomorphism is defined by the same formulas: $\sigma^{-1}\left(x^{\wedge}\right)=y, \sigma^{-1}\left(y^{\wedge}\right)=x$.

We call the algebra $H_{1 / q,-h / q}$ the conjugate to $H_{q, h}$ quantum Heisenberg algebra.

Evidently, this "conjugation" relation is reflexive. 
Now we can transfer, using the isomorphism $\sigma$, the already gathered data about left maximal ideals and representations from $H_{1 / q,-h / q}$ to $H_{q, h}$.

In particular, to the maximal left ideal $H_{1 / q,-h / q} y^{\wedge}$ and the representation $V^{\left(y^{\wedge}\right)}$ corresponds the maximal ideal $H_{q, h} x$ and the canonical representation $V^{(x)}$ of the algebra $H_{q, h}$ in the space $k[z]$ of polynomials in $z$, which is (automatically) described by the formulas:

$$
(y \cdot f)(z)=z f(z), \quad(x \cdot f)(z)=h \partial_{q} f(z),
$$

where $\partial_{q}$ is the $q$-derivation (cf. Example 2.7.4):

$$
\partial_{q} f(z)=z^{-1}(f(q z)-f(z)) /(q-1) .
$$

Thus, the $q$-derivation is, in a certain sense, conjugate to the $1 / q$-derivation.

Now consider the case when the ideal $p^{\prime}=p \cap A$ is not $\vartheta$-invariant.

3.7 Proposition. (i) Let $p^{\prime}$ be a prime ideal of the algebra A; and let there exist an element $r \in p^{\prime}$ such that $\vartheta^{-1}(r)$ does not belong to $p^{\prime}$.

Then $\mu_{r, p^{\prime}}:=A[x ; \vartheta, d] p^{\prime}+A[x ; \vartheta, d] r x$ is a left ideal of the algebra $A[x ; \vartheta, d]$, properly containing $A[x ; \vartheta, d] p^{\prime}$ and such that $\mu_{r, p^{\prime}} \cap A=p^{\prime}$.

Besides, $\mu_{r, p^{\prime}}$ is a right A-module: $\mu_{r, p^{\prime}} A=\mu_{r, p^{\prime}}$.

(ii) If the ideal $p^{\prime}$ is maximal then the left ideal $\mu_{r, p^{\prime}}$ is two-sided. The quotient algebra $A[x ; \vartheta, d] / \mu_{r, p^{\prime}}$ is naturally isomorphic to the field $A / p^{\prime}$. In particular, $\mu_{r, p^{\prime}}$ is maximal as a left (and right) ideal.

Proof. Let $r \in p^{\prime}$ but $\vartheta^{-1}(r) \notin p^{\prime}$. The ideal $A[x ; \vartheta, d] p$ is a proper subset of $\mu_{r, p^{\prime}}:=A[x ; \vartheta, d] p^{\prime}+A[x ; \vartheta, d] r x$, since the element $r x=x \vartheta^{-1}(r)-d\left(\vartheta^{-1}(r)\right)$ does not belong to $A[x ; \vartheta, d] p^{\prime}$.

(i) The intersection of the left ideal $\mu_{r, p^{\prime}}$ with $A$ coincides with $p^{\prime}$.

In fact, if it were opposite then an element $g(x)=\sum_{0 \leqq i \leqq m} x^{i} a_{i}$ could be found such that $g(x) r x \in u+A[x ; \vartheta, d] p^{\prime}$ for some element $u \in A-p^{\prime}$. Since

$$
\begin{aligned}
g(x) r x & =g(x)\left(x \vartheta^{-1}(r)-d\left(\vartheta^{-1}(r)\right)\right. \\
& =x^{m+1} \vartheta^{-1}\left(a_{m} r\right)+\sum_{0 \leqq i \leqq m-1} x^{i+1}\left(\vartheta^{-1}\left(a_{i} r\right)-d\left(\vartheta^{-1}\left(a_{i+1} r\right)\right)-d\left(\vartheta^{-1}\left(a_{0} r\right)\right)\right),
\end{aligned}
$$

the condition $g(x) r x \in A+A[x ; \vartheta, d] p^{\prime}$ can be expressed as follows:

(1) $\vartheta^{-1}\left(a_{m} r\right) \in p^{\prime}$. Since $\vartheta^{-1}\left(a_{m} r\right)=\vartheta^{-1}\left(a_{m}\right) \vartheta^{-1}(r)$ and $\vartheta^{-1}(r) \notin p^{\prime}$ by hypothesis, $\vartheta^{-1}\left(a_{m}\right) \in p^{\prime}$.

(2) $\vartheta^{-1}\left(a_{i} r\right)-d\left(\vartheta^{-1}\left(a_{i+1} r\right)\right) \in p^{\prime}$ for $0 \leqq i \leqq m-1$.

Fix $i, 0 \leqq i \leqq m-1$, and suppose that $\vartheta^{-1}\left(a_{i+1}\right) \in p^{\prime}$. Then

$$
d\left(\vartheta^{-1}\left(a_{i+1} r\right)\right)=d\left(\vartheta^{-1}(r)\right) \vartheta^{-1}\left(a_{i+1}\right)+r d\left(\vartheta^{-1}\left(a_{i+1}\right)\right) \in p^{\prime} .
$$

Therefore the condition 2) means that $\vartheta^{-1}\left(a_{i} r\right) \in p^{\prime}$, or, equivalently, $\vartheta^{-1}\left(a_{i}\right) \in p^{\prime}$.

Thus, $\vartheta^{-1}\left(a_{i}\right) \in p^{\prime}$ for $0 \leqq i \leqq m$; and, therefore, $a_{i} r x=x \vartheta^{-1}\left(a_{i} r\right)-d\left(\vartheta^{-1}\left(a_{i} r\right)\right) \in$ $x p^{\prime}+p^{\prime}$ for each $i, 0 \leqq i \leqq m$; i.e. $g(x) r x \in A[x ; \vartheta, d] p^{\prime}$.

This implies, in particular, that there are no elements $g(x)$ of the algebra $A[x ; \vartheta, d]$ such that $g(x) r x \in u+A[x ; \vartheta, d] p^{\prime}$ for some $u \in A-p^{\prime}$. 
It is easy to see that $\mu_{r, p^{\prime}}$ stands the multiplication by elements of $A$ from the right: $\mu_{r, p^{\prime}} A \subseteq \mu_{r, p^{\prime}}$ :

$$
r x a=r(\vartheta(a) x+d a)=\vartheta(a) r x+r d a \in A r x+p^{\prime} .
$$

(ii) Now suppose that the ideal $p^{\prime}$ is maximal.

The ideal $\mu_{r, p^{\prime}}$ is two-sided.

Since $\vartheta^{-1}(r) \notin p^{\prime}$ and the ideal $p^{\prime}$ is maximal, there exists an element $u$ of the algebra $A$ such that $\vartheta^{-1}(r) u \in 1+p^{\prime}$. Therefore,

$$
r x u=x \vartheta^{-1}(r) u-\gamma \in x-\gamma+x p^{\prime},
$$

where $\gamma$ denotes $u d\left(\vartheta^{-1}(r)\right)$.

Since $r x u \in \mu_{r, p^{\prime}}$ and $x p^{\prime} \subset \mu_{r, p^{\prime}}$, the inclusion (1) implies that $x-\gamma \in \mu_{r, p^{\prime}}$. In other words, the ideal $\mu_{r, p^{\prime}}$ contains the ideal

$$
v_{\gamma, p^{\prime}}:=A[x ; \vartheta, d] p^{\prime}+A[x ; \vartheta, d](x-\gamma)=p^{\prime}+A[x ; \vartheta, d](x-\gamma) .
$$

The inverse inclusion also holds, since

$$
(x-\gamma) \vartheta^{-1}(r) \in x \vartheta^{-1}(r)+d\left(\vartheta^{-1}(r)\right)+d\left(\vartheta^{-1}(r)\right) p^{\prime} .
$$

Now we can see that $\mu_{r, p^{\prime}}=v_{\gamma, p^{\prime}}$ is a right (hence two-sided) ideal, since not only $\mu_{r, p^{\prime}} A \subseteq \mu_{r, p^{\prime}}$, but also

$$
\mu_{r, p^{\prime}} x \leqq \mu_{r, p^{\prime}}(x-\gamma)+\mu_{r, p^{\prime}} \gamma \cong v_{\gamma, p^{\prime}}+\mu_{r, p^{\prime}}=\mu_{r, p^{\prime}} .
$$

Clearly the element $g(x)=\sum g_{i} x^{i}$ of the algebra $A[x ; \vartheta, d]$ belongs to the ideal $\mu_{r, p^{\prime}}=v_{\gamma, p^{\prime}}$ if and only if $g(\gamma) \in p^{\prime}$.

This implies that the map $g(x) \mapsto g(\gamma) \bmod p^{\prime}$ defines an isomorphism of the quotient algebra $A[x ; \vartheta, d] / \mu_{r, p^{\prime}}$ onto the field $A / p^{\prime}$.

3.8 Corollary. Let $p^{\prime}$ be a maximal ideal of the algebra $A$ such that $\vartheta^{-1}\left(p^{\prime}\right)-p^{\prime}$ is not empty. Then

1) The generated by $p^{\prime}$ two-sided ideal $\left[p^{\prime}\right]$ of the algebra $A[x ; \vartheta, d]$ is maximal as left (and right) ideal, For any element $r \in p^{\prime}$ such that $\vartheta^{-1}(r) \notin p^{\prime}$ the ideal $\mu_{r, p^{\prime}}:=A[x ; \vartheta, d] p^{\prime}+A[x ; \vartheta, d] r x$ coincides with $\left[p^{\prime}\right]$.

2) Let $r$ and $r^{\prime}$ be elements of the ideal $p^{\prime}$ such that $\vartheta^{-1}(r) \notin p^{\prime}$ and $\vartheta^{-1}\left(r^{\prime}\right) \notin p^{\prime}$. Then the element $\vartheta^{-1}\left(r^{\prime}\right) d\left(\vartheta^{-1}(r)\right)-\vartheta^{-1}(r) d\left(\vartheta^{-1}\left(r^{\prime}\right)\right)$ belongs to $p^{\prime}$.

Proof. 1) Clearly the ideal $\mu_{r, p^{\prime}}:=A[x ; \vartheta, d] p^{\prime}+A[x ; \vartheta, d] r x$ is contained in $\left[p^{\prime}\right]$ for any $r \in p^{\prime}$. If the element $r \in p^{\prime}$ is such that $\vartheta^{-1}(r) \in p^{\prime}$ then, by Proposition 3.7, the ideal $\mu_{r, p^{\prime}}$ is two-sided and maximal as a left (and right) ideal. In particular, $\mu_{r, p^{\prime}}$ coincides with $\left[p^{\prime}\right]$.

2) Let $\varphi$ denote the canonical epimorphism $A \rightarrow A / p^{\prime}$.

According to the first statement of this corollary, $\mu_{r, p^{\prime}}=\left[p^{\prime}\right]=\mu_{r^{\prime}, p^{\prime}}$.

On the other hand, it is clear that the ideals $\mu_{r, p^{\prime}}$ and $\mu_{r^{\prime}, p^{\prime}}$ coincide if and only if the images of $x$ with respect to the corresponding epimorphisms $A[x ; \vartheta, d] \rightarrow A / p^{\prime}$ coincide. Clearly these images are $\varphi\left(d\left(\vartheta^{-1}(r)\right)\right) / \varphi\left(\vartheta^{-1}(r)\right)$ and $\varphi\left(d\left(\vartheta^{-1}\left(r^{\prime}\right)\right)\right) / \varphi\left(\vartheta^{-1}\left(r^{\prime}\right)\right)$ respectively. They are equal each other if and only if $\vartheta^{-1}\left(r^{\prime}\right) d\left(\vartheta^{-1}(r)\right)-$ $\vartheta^{-1}(r) d\left(\vartheta^{-1}\left(r^{\prime}\right)\right) \in p^{\prime}$. 
3.9 Proposition. Let $p^{\prime}$ be a maximal ideal of the algebra $A$ such that, for any positive integer $n$, there exists an element $a \in p^{\prime}$ such that $\vartheta^{n}(a) \notin p^{\prime}$.

Then any proper left ideal of the algebra $A[x ; \vartheta, d]$, which contains $p^{\prime}$, is contained in $\left[p^{\prime}\right]$.

Proof. (i) Note that the condition $\vartheta\left(p^{\prime}\right)-p^{\prime}$ is not empty implies that $\vartheta^{-1}\left(p^{\prime}\right)-p^{\prime}$ is not empty.

Indeed, since the ideal $p^{\prime}$ is maximal, the ideal $\vartheta^{-1}\left(p^{\prime}\right)$ is also maximal. Therefore, the inclusion $\vartheta^{-1}\left(p^{\prime}\right) \subseteq p^{\prime}$ means that $\vartheta^{-1}\left(p^{\prime}\right)$ coincides with $p^{\prime}$. But then $\vartheta\left(p^{\prime}\right)=p^{\prime}$.

(ii) Let $m$ be a left ideal of the algebra $A[x ; \vartheta, d]$, which contains $p^{\prime}$. Suppose that $m$ is not a subset of $\left[p^{\prime}\right]$. Then the ideal $m$ has an element of the form $u+g(x) r x$, where $r$ is an element of $p^{\prime}$ such that $\vartheta^{-1}(r) \notin p^{\prime}$, and $u \in A-\rho^{\prime}$.

In fact, the ideal $\left[p^{\prime}\right]$ coincides with the ideal $\mu_{r, p^{\prime}}=A[x ; \vartheta, d] p^{\prime}+A[x ; \vartheta, d] r x$ (cf. the point 1) of this proof); and the ideal $\mu_{r, p^{\prime}}$ is equal to $p^{\prime}+A[x ; \vartheta, d](x-\gamma)$ for an appropriate element $\gamma \in A$ (cf. the proof of the statement (ii) of Proposition 3.7).

Every element $f=f(x)$ of the algebra $A[x ; \vartheta, d]$ can be represented in the form $f(x)=f_{1}(x)(x-\gamma)+f(\gamma)$ for some element $f_{1}(x) \in A[x ; \vartheta, d]$. Since $f_{1}(x)(x-\gamma) \in p^{\prime}+$ $A[x ; \vartheta, d](x-\gamma)=\mu_{r, p^{\prime}}$, the polynomial $f_{1}(x)(x-\gamma)$ belongs to $g(x) r x+A[x ; \vartheta, d] p^{\prime}$; and, therefore, $f(x) \in f(\gamma)+g(x) r x+A[x ; \vartheta, d] p^{\prime}$. If $f(x)$ belongs to a left ideal $m$ and $A[x ; \vartheta, d] p^{\prime} \subset m$, this inclusion means that $f(\gamma)+g(x) r x \in m$.

On the other hand, the equality $p^{\prime}+A[x ; \vartheta, d](x-\gamma)=\mu_{r, p^{\prime}}$ shows that $f(x) \notin \mu_{r, p^{\prime}}$ if and only if $f(\gamma) \in A-p^{\prime}$.

(iii) Now we need the following lemma, which, having in mind some further applications (cf. Sect. 4), we give in a slightly more general form than strictly necessary for Proposition 3.9.

3.9.1 Lemma. Let $p^{\prime}$ be a maximal ideal of the algebra A. Suppose that there exists a positive integer $N$ such that, for any $n, 1 \leqq n \leqq N$, an element $a \in p^{\prime}$ can be found with the property: $\vartheta^{n}(a) \notin p^{\prime}$.

Then for any element $g(x)$ of the ring $A[x ; \vartheta, d]$ such that $\operatorname{deg}(g(x)) \leqq N-1$ there exists an element $a \in A-p^{\prime}$, for which $\operatorname{ag}(x) r x \in A[x ; \vartheta, d] p^{\prime}$.

Proof. 1) Let $\operatorname{deg}(g)=0$; i.e. $g \in A$. By condition there exists an element $a \in p^{\prime}$ such that $\vartheta(a) \notin p^{\prime}$. We have:

$$
\vartheta(a) g r x=\operatorname{gr}(x a-d(a))=\operatorname{grx} a-d(a) r \in A[x ; \vartheta, d] p^{\prime}
$$

since $a$ and $r$ are elements of $p^{\prime}$.

2) Suppose now that the statement is true for polynomials of degree less than $n-1,2 \leqq n \leqq N$; and let $g(x)=b x^{n-1}+g_{1}(x)$, where $\operatorname{deg}\left(g_{1}\right) \leqq n-2$. By condition there exists an element $a^{\prime} \in p^{\prime}$ such that $\vartheta^{n}\left(a^{\prime}\right) \notin p^{\prime}$. Now,

$$
\vartheta^{n}\left(a^{\prime}\right) g(x) r x=b \vartheta^{n}\left(a^{\prime}\right) x^{n-1} r x+\vartheta^{n}\left(a^{\prime}\right) g_{1}(x)=b x^{n-1} \vartheta\left(a^{\prime}\right) r x+g \sim(x),
$$

where $g^{\sim}(x)=\vartheta^{n}\left(a^{\prime}\right) g_{1}(x)-\sum_{0 \leqq i \leqq n-1} x^{n-i-1} d\left(\vartheta^{i}\left(a^{\prime}\right)\right) x^{i}$.

As it has been shown in 1$), \vartheta\left(a^{\prime}\right) r x \in A[x ; \vartheta, d] p^{\prime}$. Since $\operatorname{deg}\left(g^{\sim}\right) \leqq n-2$ then, by induction hypothesis, an element $a^{\prime} \in A-p^{\prime}$ can be found such that $a^{\prime \prime} g^{\sim}(x) r x \in A[x ; \vartheta, d] p^{\prime}$. Therefore

$$
a^{\prime \prime} \vartheta^{n}\left(a^{\prime}\right) g(x) r x=a^{\prime \prime} b x^{n-1} \vartheta\left(a^{\prime}\right) r x+a^{\prime \prime} g^{\sim}(x) r x \in A[x ; \vartheta, d] p^{\prime} .
$$


It remains to notice that, since $p^{\prime} \in \operatorname{Spec} A$, the element $a^{\prime \prime} \vartheta^{n}\left(a^{\prime}\right)$ does not belong to $p^{\prime}$.

(iv) Let the left ideal $m$ contain the ideal $A[x ; \vartheta, d] p^{\prime}$ and an element of the form $u+g(x) r x$, where $u \in A-p^{\prime}$. According to the heading (iii) of this proof, there exists an element $a \in A-p^{\prime}$ such that $a g(x) r x \in A[x ; \vartheta, d] p^{\prime}$. Hence $a u=a(u+g(x) r x)-$ $a g(x) r x$ is an element of the ideal $m$. But $a u \in A-p^{\prime}$. This implies, since the ideal $p^{\prime}$ is maximal, that $m \supset A a u+p^{\prime}=A$. Therefore $m=A[x ; \vartheta, d]$.

\section{The Restricted Algebra of Skew Differential Operators and Its Left Spectrum}

4.1 Definition. Let, as before, $\vartheta$ be an automorphism of a commutative noetherian algebra $A$ and $d a$ $\vartheta$-derivation. Fix a positive integer $N$ and an element $\rho$ of the algebra $A$. With this data one can connect the restricted, or $N$-restricted, algebra $A_{N, \rho}[x ; \vartheta, d]$ of $\vartheta$-skew differential operators defined as the quotient of the algebra $A[x ; \vartheta, d]$ by the two-sided ideal generated by $x^{N}-\rho$.

Note that this definition is not so harmless as it seems to be at the first glance. In fact, since the algebra $A$ is commutative, the relation $x^{N}=\rho$ imposes, that

$$
\vartheta^{N}(a) x^{N}=x^{N} \vartheta^{N}(a) \text { for any } a \in A .
$$

On the other hand, we know from Lemma 3.1 that

$$
\vartheta^{n}(a) x^{n}=x^{n} a-\sum_{0 \leqq i \leqq n-1} x^{n-i-1} d\left(\vartheta^{i}(a)\right) x^{i}
$$

for any positive integer $n$. Therefore, if we want the powers $x, x^{2}, \ldots, x^{N-1}$ to be independent over $A$ (and we certainly do), we have to impose some additional relations; at least those, which come from the requirement of the independency and from the Eq. (1.n):

$$
\begin{gathered}
\vartheta^{N}(a)=a \text { for each } a \in A ; \text { i.e. } \vartheta^{N}=\mathrm{Id} ; \\
\sum_{0 \leqq i \leqq N-1} \vartheta^{-i} d\left(\vartheta^{i}(a)\right)=0 \text { for each } a \in A ;
\end{gathered}
$$

(cf. Propositions 3.4 and 3.5)

$$
\varphi_{i, j}(a)=0 \text { for every } a \in A, \quad 0 \leqq i \leqq j \leqq N,
$$

where the functions $\varphi_{i, j}$ are determined by the relations:

$$
\begin{aligned}
\varphi_{0,0}(a) & =a ; \quad \varphi_{i, 0}(a)=\left(-d \circ \vartheta^{-1}\right)^{i}(a), \\
\varphi_{i, i}(a) & =\varphi_{i-1, i-1}\left(\vartheta^{-1}(a)\right) \text { for } i \geqq 1 ; \\
\varphi_{i, j}(a) & =\varphi_{i-1, j-1}\left(\vartheta^{-1}(a)\right)-\varphi_{i-1, j}\left(d\left(\vartheta^{-1}(a)\right)\right)
\end{aligned}
$$

for $i \geqq 1,0 \leqq j \leqq i-1$ (cf. Proposition 3.5).

Besides, the relations $\rho x=x^{N} x=x x^{N}=x \rho=\vartheta(\rho) x+d(\rho)$ and the requirement " $x$ is a non-zero divisor" imply that $\rho$ is a fixed "point" of the automorphism $\vartheta$ and of the $\vartheta$-derivative $d: \vartheta(\rho)=\rho, d(\rho)=0$. All together these conditions are equivalent to the following one:

(*) the elements $x^{N}$ and $\rho$ of the algebra $A[x ; \vartheta, d]$ are central. 
4.2 Lemma. Suppose that the relations (1)-(3) hold, and $\vartheta(\rho)=\rho$. Then the elements $1, x, x^{2}, \ldots, x^{N-1}$ form a basis of $A_{N, \rho}[x ; \vartheta, d]$, considered as a right or left $A$-module.

Proof. In fact, since the elements $x^{N}$ and $\rho$ are central, the determining algebra $A_{N, \rho}[x ; \vartheta, d]$ (in $A[x ; \vartheta, d]$ ) two-sided ideal is a left (and right) principal ideal, generated by $x^{N}-\rho$. The equality $\sum_{1 \leqq i \leqq n} a_{i} x^{i}=0$ in $A_{N, \rho}[x ; \vartheta, d]$ means that the polynomial (an element of $A[x ; \vartheta, d]) \quad g(x)=\sum_{1 \leqq i \leqq n} a_{i} x^{i}$ belongs to the ideal $A[x ; \vartheta, d]\left(x^{N}-\rho\right)$. This implies that $g(x) \equiv 0$ if $\operatorname{deg}(g) \leqq N-1$.

Further on we will include in the notion of $N$-restricted algebra $A_{N, \rho}[x ; \vartheta, d]$ of skew differential operators the requirement $\vartheta^{N}=\mathrm{Id}$ and the rest of conditions of Lemma 4.2 (i.e. the condition $(*)$ above).

4.3 Example. Restricted Quantum Heisenberg Algebras. Let now $H_{q, h}=H_{q, h}[x]$ be a quantum Heisenberg algebra, $q, h \in k$. By definition, a necessary condition for the existence of $N$-restricted quantum Heisenberg algebras is $q$ being a $N^{\text {th }}$ root of unity: $q^{N}=1$. This condition is also sufficient.

Indeed, $y \cdot x^{n}=q^{-n} x^{n} y-x^{n-1} h\left(1-q^{-n}\right) /(1-q)$ for any integer $n \geqq \perp$ (cf. 3.6). In particular, $y x^{N}=x^{N} y$ if $q^{N}=1$. Therefore the monom $x^{N}$ belongs to the center of the algebra $H_{q, h}$.

Suppose that $q^{N}=1$, but $q^{n} \neq 1$ for $0 \leqq n \leqq N-1$. Clearly an element $\rho$ of the algebra $k[y]$ is $q_{*}$-invariant if and only if it is a polynomial in $y^{N}$. If $q \neq 1$ and $h \neq 0$, then the polynomial $f(y)$ is $q_{*}$-invariant if and only if $d(f) \equiv 0$. If $q=1$ (and $h \neq 0$ ) then $d(f) \equiv 0$ if and only if $\operatorname{deg}(f)=0$.

4.4 Restriction by Changing the Base. We have introduced the restricted algebras of $\vartheta$-skew differential operators to study the spectrum and irreducible representations of non-restricted algebras $A[x ; \vartheta, d]$ in case when $\vartheta^{N}=$ Id for some $N$. The corresponding reduction is made as follows.

Let the algebra $A[x ; \vartheta, d]$ admit a $N$-restriction; i.e. $\vartheta^{N}=$ Id and the element $x^{N}$ belongs to the center of $A[x ; \vartheta, d]$. In particular, the generated by $A$ and $x^{N}$ subalgebra $B:=A\left[x^{N}\right]$ of the algebra $A[x ; \vartheta, d]$ is commutative. Denote by $\vartheta^{\sim}$ and by $d^{\sim}$ the trivial extensions on the algebra $B$ of the automorphism $\vartheta$ and of the $\vartheta$-derivation $d$ respectively: $\vartheta^{\sim}\left(x^{N}\right)=x^{N}, d^{\sim}\left(x^{N}\right)=0$. Finally, take $\rho:=x^{N} \in B$.

Then, clearly, the algebra $A[x ; \vartheta, d]$ coincides with the $N$-restricted algebra $B_{N, \rho}\left[x ; \vartheta^{\sim}, d^{\sim}\right]$.

4.5 The Left Spectrum of the Algebra $A_{N, \rho}[x: \vartheta, d]$. We suppose that the algebra $A$ is noetherian and the conditions of Lemma 4.2 hold; i.e. $\vartheta^{N}=\mathrm{Id}$, and $x^{N}, \rho$ are central elements of the algebra $A_{N, \rho}[x ; \vartheta, d]$. Thanks to the noetherian condition, we can (and will) take into consideration only those $p \in \operatorname{Spec}_{l} A_{N, \rho}[x ; \vartheta, d]$, for which the intersection $p \cap A$ belongs to $\operatorname{Spec} A$.

There are two possibilities for an ideal $p \in \operatorname{Spec}_{l} A_{N, \rho}[x ; \vartheta, d]$ : either (a) $p \cap A \neq 0$, or (b) $p \cap A=0$. Consider each of them.

(a) The set of all the left ideals $p$ from $\operatorname{Spec}_{l} A_{N, \rho}[x ; \vartheta, d]$ such that $p \cap A$ is a nonzero prime ideal of $A$, is described by the straightforward analogues of Propositions 3.4, 3.5, 3.7 and 3.9, which we formulate here for the convenience of the reader. 
4.5.1 Proposition. Suppose that $p^{\prime}$ is a prime ideal of the algebra $A$ satisfying the following conditions:

(a) $p^{\prime}$ is invariant with respect to $\vartheta$;

(b) for any integer $n$ such that $1 \leqq n \leqq N-1$, there exists an element $r \in p^{\prime}$ such that

$$
\sum_{0 \leqq i \leqq n-1} \vartheta^{-i} d\left(\vartheta^{i}(r)\right) \notin p^{\prime}
$$

Then

1) The left ideal $p=A_{N, \rho}[x ; \vartheta, d] p^{\prime}$ belongs to the left spectrum of the algebra $A_{N, \rho}[x ; \vartheta, d]$.

2) The left ideal $p=A_{N, \rho}[x ; \vartheta, d] p^{\prime}$ is maximal if and only if the ideal $p^{\prime}$ is maximal.

Proof is a repetition of the proof of Proposition 3.4 .

4.5.2 Proposition. Let $p^{\prime}$ be a prime ideal of the algebra $A$ satisfying the conditions of Proposition 4.5.1. Then

1) The quotient module $V^{p^{\prime}}:=A_{N, \rho}[x ; \vartheta, d] / p$ is the direct sum of its $k$-submodules $V_{i}=x^{i} V_{0}, 0 \leqq i \leqq N-1$, where $V_{0}$ is the image of $A$ with respect to the canonical epimorphism $A_{N, \rho}[x ; \vartheta, d] \rightarrow V^{p^{\prime}}$.

The action of $A_{N, \rho}[x ; \vartheta, d]$ is given by the formulas:

$$
x \cdot\left(x^{i} v\right)=x^{i+1} v ; \quad a \cdot\left(x^{i} v\right)=\sum_{0 \leqq j \leqq i} x^{j} \varphi_{i, j}(a) \cdot v
$$

for any $v \in V_{0}, a \in A$ and $1 \leqq i \leqq N-1$, where the functions $\varphi_{i, j}$ (from $A$ to $A$ ) are determined by the relations:

$$
\begin{aligned}
\varphi_{0,0}(a) & =a ; \quad \varphi_{i, 0}(a)=\left(-d \circ \vartheta^{-1}\right)^{i}(a), \\
\varphi_{i, i}(a) & =\varphi_{i-1, i-1}\left(\vartheta^{-1}(a)\right) \quad \text { for } \quad i \geqq 1 \\
\varphi_{i, j}(a) & =\varphi_{i-1, j-1}\left(\vartheta^{-1}(a)\right)-\varphi_{i-1, j}\left(d\left(\vartheta^{-1}(a)\right)\right)
\end{aligned}
$$

for $i \geqq 1,0 \leqq j \leqq i-1$.

2) The left ideal $p=A_{N, \rho}[x ; \vartheta, d] p^{\prime}$ is maximal if and only if the ideal $p^{\prime}$ is maximal.

Proof follows the argument of the proof of Proposition 3.5.

Denote by $B$ the algebra $A_{N, \rho}[x ; \vartheta, d]$.

4.5.3 Proposition. Let $p^{\prime}$ be a prime ideal of the algebra $A$; and let there exist an element $r \in p^{\prime}$ such that $\vartheta^{-1}(r)$ does not belong to $p^{\prime}$. Then

(i) The left ideal $\mu_{r, p^{\prime}}:=B p^{\prime}+B r x$ of the algebra $B$ contains properly the left ideal $B p^{\prime}$ and $\mu_{r, p^{\prime}} \cap A=p^{\prime}$.

Besides, $\mu_{r, p^{\prime}}$ is a right $A$-module: $\mu_{r, p^{\prime}} A=\mu_{r, p^{\prime}}$.

(ii) If the ideal $p^{\prime}$ is maximal then the left ideal $\mu_{r, p^{\prime}}$ is two-sided. The quotient algebra $B / \mu_{r, p^{\prime}}$ is naturally isomorphic to the field $A / p^{\prime}$. In particular, $\mu_{r, p^{\prime}}$ is maximal as a left (and right) ideal.

4.5.4 Proposition. Let $p^{\prime}$ be a maximal ideal of the algebra $A$ such that $\vartheta^{n}\left(p^{\prime}\right)$ is not a subset of $p^{\prime}$, for any positive integer $n, 1 \leqq n \leqq N-1$. 
Then any proper left ideal of the algebra $A_{N, \rho}[x ; \vartheta, d]$, which contains $p^{\prime}$, is contained in the two-sided ideal $\left[p^{\prime}\right]$ generated by $p^{\prime}$.

(b) Consider now the set of the left ideals $p$ from $\operatorname{Spec}_{l} A_{N, \rho}[x ; \vartheta, d]$ such that $p \cap A=0$.

Since the ideal $p \cap A$ is prime, the condition $p \cap A=0$ implies that $A$ is a prime ring. The localization $Q$ at the set $A \backslash\{0\}$ sends the algebra $A_{N, \rho}[x ; \vartheta, d]$ into the algebra $Q A_{N, \rho}[x ; \vartheta, d]$ over the skew field of quotients $Q A$, and the ideal $p$ into the left ideal $Q p$ from $\operatorname{Spec}_{l} Q A_{N, \rho}[x ; \vartheta, d]$.

According to Lemma $4.2, Q A_{N, \rho}[x ; \vartheta, d]$ is a $N$-dimensional algebra over the field $Q A$. Again we have two possibilities: $\rho=0$ and $\rho \neq 0$.

4.5.5 Lemma. Let $\rho=0$; i.e. $x^{N}=0$. Then any left ideal from $\operatorname{Spec}_{l} Q A_{N, \rho}[x ; \vartheta, d]$ is equivalent to $Q A_{N, \rho}[x ; \vartheta, d] x$.

Proof. The left ideals from $\operatorname{Spec}_{l} Q A_{N, \rho}[x ; \vartheta, d]$ are exactly the left maximal (principal) ideals $Q A[x ; \vartheta, d] r$, which contain $x^{N}$; i.e. $x^{N}=f(x) r(x)$. Clearly this implies that the irreducible element $r(x)$ of the algebra $Q A[x ; \vartheta, d]$ is equivalent to $x$.

4.5.6 Lemma. Let $x^{N}$ and $\rho \in A \backslash\{0\}$ be central elements of the algebra $A[x ; \vartheta, d]$. Then the set of all the maximal left ideals of the algebra $A_{N, \rho}[x ; \vartheta, d]$ strictly containing $x^{N}-\rho$, consists of

$\alpha$ ) all the (two-sided) ideals, $m_{\gamma}:=m+A[x, \vartheta, d](x-\gamma)$, where $m \in \operatorname{Max} A, \rho \notin m$, and $\gamma \in A$ is such that $\gamma^{N}-\rho \in m$ and $\gamma(a-\vartheta(a))-d(a) \in m$ for every $a \in m$;

$\beta)$ the left ideals $A[x ; \vartheta, d] \cap Q A[x ; \vartheta, d] r$, where $r=r(x)$ is an element of $A[x ; \vartheta, d]$, which is an irreducible element of the algebra $Q A[x ; \vartheta, d]$ such that

B.1) $x^{N}-\rho=g(x) r(x)$ for some $g(x) \in Q A[x ; \vartheta, d] \backslash Q A$;

B.2) $r(\gamma) \notin m$ for every $m \in \operatorname{Max} A$ and every $\gamma \in A \backslash m$ such that $\gamma(a-\vartheta(a))-d(a) \in m$ for every $a \in m$.

Proof.

(1) Suppose that $x^{N}-\rho$ is not an irreducible element of the algebra $Q A[x ; \vartheta, d]$. Then $x^{N}-\rho=f(x) r(x)$, where $r(x) \in A[x ; \vartheta, d]$ is an irreducible element of the algebra $Q A[x ; \vartheta, d]$ such that $\operatorname{deg}(r(x))<N$.

Denote by $m\langle r\rangle$ the preimage of the ideal $Q A[x ; \vartheta, d] r$ in $A[x ; \vartheta, d]$. There are, as usual, two possibilities; either $m\langle r\rangle$ is a maximal left ideal or not.

If not, then the ideal $m\langle r\rangle$ is contained in some maximal left ideal $\mu$ of the algebra $A[x, \vartheta, d]$ such that $\mu \cap A$ is a maximal ideal of the algebra $A$.

If the ideal $\mu \cap A$ were $\vartheta$-invariant then, by Proposition 4.5.1, $\mu=A[x ; \vartheta, d](\mu \cap A)$. In particular, the element $x^{N}-\rho$ belongs to $A[x ; \vartheta, d](\mu \cap A)$. But this is, obviously; not the case.

Hence the ideal $\mu \cap A$ is not $\vartheta$-invariant. Then, by Proposition 3.7, there exists $\gamma \in A \backslash \mu$ such that

$$
\mu=(\mu \cap A)+A[x ; \vartheta, d](x-\gamma) .
$$

Or, in other words, the ideal $\mu$ consists of all the $f(x) \in A[x ; \vartheta, d]$ such that $f(\gamma) \in \mu \cap A$. As a sequence, taking $f(x)=x^{N}-\rho$, we obtain the relation: $\gamma^{N}-\rho \in \mu \cap A$. 
4.5.6.1 Sublemma. Let $m$ be a maximal ideal of the algebra $A$ such that $\rho \notin m$, and there exists $\gamma \in A$, for which

$$
\gamma(a-\vartheta(a))-d(a) \in m \text { for any } a \in m .
$$

Then

(i) $m_{\gamma}:=m+A[x ; \vartheta, d](x-\gamma)=\{f(x) \in A[x ; \vartheta, d]: f(\gamma) \in m\}$ is a two-sided ideal in $A[x ; \vartheta, d]$, which is maximal as a left (and right) ideal;

(ii) The following conditions are equivalent:

a) the ideal $m_{\gamma}$ contains $x^{N}-\rho$;

b) $\gamma^{N}-\rho \in m$ for some $\gamma$, satisfying the condition (1);

c) $\gamma^{N}-\rho \in m$ for any $\gamma$, satisfying the condition (1).

Proof. For any maximal ideal $m$ of the algebra $A$ and an element $\gamma \in A$, the left ideal $m_{\gamma}$ is proper if and only if $\gamma(a-\vartheta(a))-d(a) \in m$ for any $a \in m$. It is easy to see that if the ideal $m_{\gamma}$ is proper then it is maximal as a left ideal.

If $\gamma, \gamma^{\sim}$ are elements of $A$ such that $\gamma(a-\vartheta(a))-d(a) \in m$ and $\gamma^{\sim}(a-\vartheta(a))-d(a) \in m$, then $\left(\gamma-\gamma^{\sim}\right)(a-\vartheta(a)) \in m$ for any $a \in m$. Since, by condition, the ideal $m$ is not $\vartheta$-invariant, $a-\vartheta(a)$ does not belong to the ideal $m$ for some $a \in m$. This implies, since the ideal $m$ is prime, that $\gamma-\tilde{\gamma}^{\tilde{\gamma}} \in m$.

Conversely, if $\gamma(a-\vartheta(a))-d(a) \in m$ and $\tilde{\gamma}$ is an element of $A$ such that $\gamma-\tilde{\gamma} \in m$, then $\gamma^{\sim}(a-\vartheta(a))-d(a) \in m$.

We know from Proposition 3.7 that the ideal $m_{\gamma}$ is two-sided. This and the considerations above imply that $m_{\gamma}=m_{\gamma^{*}}$ if $\gamma-\gamma^{\sim} \in m$. It follows also from the equality

$$
m_{\gamma}=\{f(x) \in A[x ; \vartheta, d]: f(\gamma) \in m .
$$

Now the equivalence of the conditions a), b), c) in (ii) follows immediately.

Thus, if the element $x^{N}-\rho$ is reducible then the set of the left maximal ideals containing $x^{N}-\rho$ consists of

$\alpha$ ) two-sided maximal ideals, $m_{\gamma}:=m+A[x ; \vartheta, d](x-\gamma)$, where $m \in \operatorname{Max} A$, $\rho \notin m$, and $\gamma \in A$ is such that $\gamma^{N}-\rho \in m$ and $\gamma(a-\vartheta(a))-d(a) \in m$ for every $a \in m$;

$\beta$ ) the left ideals $A[x ; \vartheta, d] \cap Q A[x ; \vartheta, d] r$, where $r=r(x)$ is an element of $A[x ; \vartheta, d]$, which is an irreducible element of the algebra $Q A[x ; \vartheta, d]$ such that

ß.1) $x^{N}-\rho=g(x) r(x)$ for some $g(x) \in Q A[x ; \vartheta, d]$;

$\beta$.2) $r(\gamma) \notin m$ for every $m \in \operatorname{Max} A$ and every $\gamma \in A \backslash m$ such that $\gamma(a-\vartheta(a))-d(a) \in m$ for every $a \in m$.

(2) Let now $x^{N}-\rho$ be an irreducible element of the algebra $Q A[x ; \vartheta, d]$. Then the set of the maximal left ideals, strictly containing $x^{N}-\rho$, consists of all the two-sided ideals

$$
m_{\gamma}:=m+A[x ; \vartheta, d](x-\gamma)=\{f(x) \in A[x ; \vartheta, d]: f(\gamma) \in m\},
$$

where $m \in \operatorname{Max} A, \rho \notin m$, and $\gamma \in A$ is such that $\gamma^{N}-\rho \in m, \gamma(a-\vartheta(a))-d(a) \in m$ for every $a \in m$.

Acknowledgements. I am indebted to David Kazhdan for provoking me to study the quantum Heisenberg algebra and for stimulating discussions. I would like also to thank Nikolai Reshetikhin for valuable suggestions. 


\section{References}

[C] Connes, A.: Géométrie Noncommutative. Paris: InterEditions 1990

[D] Dixmier, J.: Algèbres Enveloppantes. Paris-Bruxelles-Montreal: Gauthier-Villars 1974

[Dr] Drinfeld, V. G.: Quantum Groups. Proc. Int. Cong. Math., pp. 798-820. New York: Berkeley 1986

[FG] Gelfand, I. M., Fairlie, D. B.: The Algebra of Weyl Symmetrized Polynomials and its Quantum Extension. Commun. Math. Phys. 13 (1991)

[FK] Frenkel, I. B., Kac, V. G.: Basic Representations of Affine Lie Algebra and Dual Resonance Models, Invent. Math. 62, 23-66 (1980)

[FRT] Faddeev, L., Reshetikhin, N., Takhtajan, L.: Quantization of Lie Groups and Lie Algebras, preprint, LOMI-14-87; Algebra Analysis 1, no. 1 (1989)

[J] Jimbo, M.: A $q$-Difference Analog of $U(\mathfrak{g})$ and the Yang-Baxter Equation. Lett. Math. Phys. 10, 63-69 (1985)

[JG] Jaffe, A., Glimm, J.: Quantum Physics. Berlin, Heidelberg, New York: Springer 1987

[K] Kirillov, A.: Unitary representations of Unipotent Groups. Russ. Math. Surv. 17, 57-110 (1962)

[M] Manin, Yu. I.: Quantum Groups and Non-commutative Geometry, CRM, Université de Montréal (1988)

[R1] Rosenberg, A. L.: Noncommutative Affine Semischemes and Schemes, 'Seminar on Supermanifolds,' vol. 26, pp. 1-317. Reports of Dept. of Math. of Stockholm University 1988

[R2] Rosenberg, A. L.: Left Spectrum, Levitzki Radical and Noncommutative Schemes, Proc. of Natl. Acad., vol. 87, 1990

[S] Smith, S. P.: Quantum Groups: An Introduction and Survey for Ring Theorists, preprint

Communicated by N. Yu. Reshetikhin 